\title{
On a Strategy of Mobile Networks Synthesis
}

\author{
VLADIMIR LYANDRES \\ School of Electrical and Computer Engineering, \\ Ben-Gurion University of the Negev, \\ P.O. Box 653, Beer-Sheva, 84105 \\ ISRAEL
}

\begin{abstract}
Effective design of mobile communication network includes optimization of two bounded together processes: the network base stations placement and the channel assignment. In real environments the well-known cellular concept fails due to not uniformly spaced traffic and not isotropic wave propagation. We find a rather universal method for synthesis of a close to optimal network structure. The proposed design approach is based on the idea of adaptive vector quantization for a map with random traffic. As a result, the service zone of the network becomes discrete and is being transformed to a map with not equal cells and approximately equal number of requests in every one. This fact allows to apply the simplest periodic reuse pattern. The algorithm finds a point with minimal average Euclidean distance from all its requests. This point defines optimal placement of the corresponding base station. The approach guarantees maximum coverage.
\end{abstract}

Key-words: Communications, Mobile networks design, Vector quantization

Received: February 2, 2021. Revised: July 23, 2021. Accepted: July 28, 2021. Published: August 9, 2021.

\section{Introduction}

Mobile radio networks operating nowadays have been designed with the help of the wellknown cellular concept. It considers the space structure of the network as a regular hexagonal cell lattice with a periodic frequency reuse pattern $[1,2]$. This design strategy is widely accepted as it provides the most economic covering of the service area, the densest packing of co-channel cells and is relatively simple. Except for cell splitting [3], no other theoretical solution has been proposed to overcome those events.

The frequency assignment problem (FAP) has been extensively studied, and many heuristics were proposed to solve it effectively [4-7]. Since most of these techniques deal with fixed distance and/or frequency constraints they "generate" regular reuse patterns, i.e. solve FAP for networks built following classical cellular concept. However, it does not permit to fulfill design that is adapted to not homogeneous and not isotropic radio wave propagation, as well as to not constant or a priori not known traffic density (distribution of channel requests). The latter point is the most significant factor in the complexity of the efficient network design [8], which must answer the following requirements: it must be capable to find the optimal location of base stations according to the spatial users distribution; it must be capable to find the optimal location of base stations according to the spatial users distribution, it must take into account the propagation conditions to guarantee maximum service coverage, and at last, it must create configurations as homogeneous as possible in order to reduce the complexity of FAP solution.

A number of studies aimed to optimize the network design is known, however, most of them were focused in providing coverage requirements, without paying much attention to the FAP complexity [9-11]. The adaptive traffic design approach leads to a not regular network structure without fixed reuse pattern and requires a signal-to interference (SIR) test after the FAP is solved. What is not less important, overestimation of constraints may waste spectrum.

It would be desirable to find such a technique for the FAP that performs the SIR tests in the assignment process itself, minimizes spectrum 
and provides the desirable high homogeneity of the network.

\section{Vector Quantization}

Our problem has certain analogy to a wellknown problem in information theory, namely, so called vector quantization (VQ) [12], that involves classification of data blocks into a discrete number of cells in such a way that optimizes some quality criterion, for example mea square distortion. It represents an evident extension of scalar quantization, which includes two operations over continuous-time and continues-amplitude signal: sampling and quantization. This converts the signal into a sequence of discrete-time quantized values. VQ is characterized by its dimension, i.e. by the number of joint samples, which is considered as a single vector. Then, VQ approximates an infinite set of source vectors by a limited set of code vectors (in the scalar case by a limited set of discrete amplitudes), that forms a code book. The evident distortion, which takes place in such representation of a multidimensional signal is measured by a cost function, commonly the squared Euclidean norm or mean square error. In the framework of mobile network design, the source (signal) is the two dimensional field with spatially distributed channel requirements.

The VQ problem is formulated as following: given the source vector $x$ with probability multidimensional density $f(x)$, the code vector $y_{i}$ and the mean square error

$d^{2}(x, y)=\sum_{j=1}^{k}\left|x_{j}-y_{\mathrm{ij}}\right|^{2}, i \in\{1, \ldots, N\}$

The aim is to find optimal code vector which minimizes

$$
D^{2}=E\left[d^{2}\left(x, y_{i}\right)\right], \forall x
$$

The code book is then partitioned in such a way that for each vector $x$ a nearest neighbor code vector $y_{i}$ exists. This operation is known as a Voronoi partition, with the code vectors being the centroids of each Voronoi region.

In our case we may write out the VQ problem is formulated in such a way:

given a set $S$ of $n$ points in $R^{2}$; to find its Voronoi partition, i.e. to break down if $R^{2}$ into $n$ two-dimensional regions vo $(p)$ where $p \in S$, which are called the Voronoi cells of $\mathbf{p}$, and are defined as the set of points in $R^{2}$ that are closer to any other points in $S$, or more precisely:

$$
\begin{aligned}
& \operatorname{vo}(p)=\left\{x \in R^{2} \mid \operatorname{dist}(x, p) \leq\right. \\
& \operatorname{dist}(x, q) \forall q \in(S-p) \mid\}
\end{aligned}
$$

The dist in (3) is the Euclidean distance function. The set of all such cells forms a cell complex with so called Voronoi vertices.

\section{Vector quantizer design problem with help of training sequence}

In general, the probability density function of the source is rarely known and what's more it is not stationary. The rate distortion theory offers for overcoming this difficulty to use a training sequence that best represents the source in order to optimize the code book by applying a clustering Lloyd algorithm [13]. However, in solution of adaptive VQ it needs certain modifications. If in the clustering analysis one wishes to group things, and the groups can change in time, in VQ one wishes to fix the group in order to get a time-invariant quantizer and then use it on future data outside the training sequence.

The problem of design adaptive vector quantizer design can be stated as follows:

given a vector source with certain statistical parameters, given a distortion measure, given a training sequence consisting of $M$ twodimensional source vectors

$$
X=\left\{x_{1}, x_{2}, \ldots, x_{M}\right\}
$$

Find a code book and a partition which result together in the smallest average distortion. We assume that the training sequence is sufficiently long, so that all the statistical properties of the source are captured by the training sequence.

Let $N$ be the number of code vectors forming the code book:

$$
C=\left\{y_{1}, y_{2}, \ldots, y_{N}\right\}
$$

Let $S_{n}$ be the encoding rejoin associated with code vector

$y_{n}$ and let us denote the partition of the space as

$$
S=\left\{S_{1}, S_{2}, \ldots, S_{N}\right\}
$$

If the source vector $x_{m}$ is in the encoding rejoin $S_{n}$, then its approximation, denoted by $q\left(x_{m}\right)$ is $y_{n}$, i.e.

$$
q(x)=y_{n} \forall x \in S_{n}
$$


Averaging a square distance measure, we obtain:

$$
\bar{D}=\frac{\sum_{m=1}^{M}\left\|x_{m}-q\left(x_{m}\right)\right\|^{2}}{M}
$$

In nomenclature of optimization theory, our problem can be stated as follows: given $X$ and $N$, find $C$ and $S$, such that $\bar{D}$ is minimized. If $C$ and $S$ represent together a solution, then they must satisfy two criteria of optimality: nearest neighbor condition and centroid condition. The first criterion requires the following: the encoding region $S_{n}$ should consist of all vectors that are closer to $y_{n}$ than any of the other code vectors, i.e.

$\left.y_{n}\left\|^{2} \leq\right\| x-y_{l} \|^{2} \forall l \neq n\right\}$

$$
S_{n}=\{x: \| x-
$$

(9)

This expression is practically the same as (3), meaning that the optimal partition is a Voronoi partition.

The second criterion requires from the code vectors to be average of all training vectors that are in the encoding region.

A solution fulfilling to these two conditions of optimal VQ for a known probabilistic model or on a long training sequence of data may be obtained with help of the Linde-Buzo-Gray (LBG) algorithm [14], with the most known application in speech processing.

\section{VQ as a mobile network design strategy}

It is almost evident that the VQ problem has some points in common with the problem of effective mobile network design, so it is worth to start by redefining all variables of VQ in the frame work of network design.

A two-dimensional source with known longterm statistical behavior properties is a map with the spatially distributed traffic. Traditionally, this information is represented by the data from so-called service test points (STP), which are defined on a grid with available propagation and service information. However, if in the case of speech processing those STPs may be used themselves as a training sequence in the LBG algorithm, in the network design problem samples of the real space traffic distribution obtained with help of so-called demand mode concept (DMC) are preferable [15]. In this approach, a demand node represents the center of the area that contains a quantum of traffic, i.e. a fixed number of call requests per time unit, so the DMC discretizes the traffic distribution in both space and demand. This empirical data for every area of the map is derived from population distribution and is to be stored in a traffic matrix. The demand nodes are dense in highly populated areas and sparse in less populated regions. An alternative approach to create training sequences with the advantage of mathematical tractability is to model the spatial traffic distribution with an analytical bi-variate probability density function and perform a Monte-Carlo process that could create a sampled version of the analytical expression.

The code book of two-dimensional VQ is directly mapped to the set of the network base stations. Finding the optimal code book is equivalent to finding their optimal location. A problem arises when we are going to design a completely new network with predetermined number of base stations. In those cases, the classical cellular concept approach may be applied to generate their optimized set. A network with a regular hexagonal lattice now can be regarded as the equivalent of a uniform quantizer and the corresponding set of base stations as the equivalent of the initial code book to be optimized.

The Euclidean distance as the criteria of optimization is adequate to the case of macro cellular environment where the path loss mainly depends on the distance between transmitter and receiver, increasing according to an exponential rule. It is worth to clarify here that this distortion measure represents a distance excess and therefore an increase of transmitting power and interference to neighbor cells. Hence, minimizing the Euclidean distance means minimization of path loss excess and so optimization of transmitting power.

According to equation (3) the Voronoi partition guaranties that for any demand of service generated within the covered area, the closest base station will carry it. Under the assumption of propagation conditions defined as a function of only distance, the coverage of the furthest point from a base station within the Voronoi region would guarantee coverage of all the rest. Since the goal of VQ is to achieve a local uniformity of the source probability within every encoding region, the same local uniformity is expected in the network synthesized. This means that in every cell the probability of finding the training sequence of 
LBG algorithm is slightly the same, which is clear from consideration the fact that in a Voronoi cell complex a higher concentration of training vectors is found in smaller cells.

\section{Conclusion}

We consider an approach to effective deign of mobile network with not uniform space distribution of requests. It is based on the idea of vector quantization and fulfills all the requirements identified as necessary for adaptation to traffic, i.e. guarantees the optimal location of base stations as well as maximum coverage. The proposed method crates configurations as homogeneous as possible in order to reduce the complexity of the frequency-assignment. Such homogeneity can be achieved by adapting the size of every cell to the user distribution in such a way that the approximately the same number of users can be expected within every cell, leading to the same number of channel requests.

\section{References}

[1] McDonald V. H. The Cellular Concept. The Bell System Technical Journal. 1979, vol. 58, no. 1, pp. 15-41.

[2] Lee W. C. Y. Elements of Cellular Mobile Radio Systems. IEEE Transaction on Vehicular Technology, 1986, vol. 35, no.2, pp. 48-56.

[3] Stallings W. Wireless Communications and Networks. 2-nd ed. Prentice Hall. 2005. pp. 596.

[4] Box F., A Heuristic Technique for Assigning Frequencies to Mobile Radio Nets, IEEE Transactions on Vehicular Technology, 1979, vol. 27, pp. 57-64.

[5] Baybars I. Optimal Assignment of Broadcasting Frequencies. European Journal of Operations Research, 1982, no. 9, pp. 257263.

[6] Borndörfer R., Eisenblätter A., Grötschel M., \& Martin A. Frequency Assignment in Cellular Phone Networks. Annals of Operations Research, 1998, no. 76, pp.73-93. [7] Lyandres V., Heuristics of Channel Allocation in Radio Networks, Information and Control Systems, 2016, no. 3, pp. 47-50.

[8] Aardal, K. I., Hurkens, C. A. J., Lenstra, J. K., Tiourine, S. R. Algorithms for Radio Link Frequency Assignment: The CALMA project. Operations Research, 2002, vol. 50, no. 6 , pp. $968-980$.
[9] Chavez-Santiago R., Gigi E., Lyandres V., Complexity Analysis of a Heuristic Method for Fixed-Frequency Assignment Including Adjacent Channel Interference, IEEE Transactions on Electromagnetic Compatibility, 2008, vol. 50, no. 1, pp. 203208.

[10] Hurley S., Planning Effective Cellular Mobile Radio Networks, IEEE Transactions on Vehicular Technology, 2002, vol. 51, no. 2, pp. 48-56.

[11] Calegarie P., Guidec F., Kuonen P., Chamaret B., Udeba S., Josselin S., Wagner D., Radio Network Planning with Combinatorial Algorithms, ACTS Mobile Communications., 1996, pp. 707-713.

[12] Proakis J. G., Digital Communications, $3^{\text {rd }}$ ed., 1995, McGraw-Hill, pp. 928.

[13] Aurenhammer F., Voronoi Diagrams -A Survey of a Fundamental Geometric Data Structure. 1991, ACM Computing Surveys, vol. 23, no. 3, pp. 345-405.

[14] Lloyd S., Least Squares Quantization in PCM, IEEE Transactions on Information Theory, 1982, vol. 28, no. 2, pp. 129-137.

[15] Tutscku K., Tran-Gia P., Spacial Traffic Estimation and Characterization for Mobile Communication Network Design. IEEE Journal of Selected Areas in Communications, 1998, vol. 16, no. 5, pp. 804-811.

\section{Creative Commons Attribution License 4.0 (Attribution 4.0 International, CC BY 4.0)}

This article is published under the terms of the Creative Commons Attribution License 4.0

https://creativecommons.org/licenses/by/4.0/deed.en US 